\title{
Pattern-Based Algorithm for Peptide Sequencing from Tandem High Energy Collision-Induced Dissociation Mass Spectra
}

\author{
Wade M. Hines, Arnold M. Falick, Alma L. Burlingame, and \\ Bradford W. Gibson \\ Department of Pharmaceutical Chemistry, University of California at San Francisco, \\ San Francisco, California, USA
}

\begin{abstract}
A new strategy is reported for extracting complete and partial sequence information from collision-induced dissociation (CID) spectra of peptides. CID spectra are obtained from high energy CID of peptide molecular ions on a four-sector tandem mass spectrometer with an electro-optically coupled microchannel array detector. A peak detection routine reduces the spectrum to a list of peak masses and peak heights, which is then used for sequencing. The sequencing algorithm was designed to use spectral data to generate sequence fits directly rather than to use data to test the fit of series of sequence guesses. The peptide sequencing algorithm uses a pattern based on the polymeric nature of peptides to classify spectral peaks into sets that are related in a sequence-independent manner. It then establishes sequence relationships among these sets. Peak detection from raw data takes $10-20 \mathrm{~s}$, with sequence generation requiring an additional 10-60 s on a Sun 3/60 workstation. The program is written in the $C$ language to run on a Unix platform. The principal advantages of our method are in the speed of analysis and the potential for identifying modified or rare amino acids. The algorithm was designed to permit real-time sequencing but awaits hardware modifications to allow real-time access to CID spectra. (f Am Soc Mass Spectrom 1992, 3, 326-336)
\end{abstract}

$\mathrm{P}$ rotein sequence analysis plays an integral role in protein chemistry. It is used to give molecular identity to unknown proteins, such as a spot from a two-dimensional gel [1] or an isolated fraction with a specific activity. It can provide short sequences for the construction of oligonucleotide probes for gene cloning $[2,3]$ and can verify the structure of expressed proteins [4]. Such analysis not only discovers information with intrinsic value, but is also a prerequisite to further higher order structural characterization by such techniques as $x$-ray crystallography or nuclear magnetic resonance. Mass spectrometrists have long been interested in protein sequence analysis [5], but recently researchers interested in peptides and proteins have become interested in mass spectrometry as an alternative means for analyzing peptide primary structure. This new interest goes beyond the vital role mass spectrometry has played in the analysis of posttranslationally modified proteins [6] and can, in part,

Address reprint requests to Bradford $W$. Gibson, Department of Pharmaceutical Chemistry, University of California at San Francisco, San Francisco, CA 94143-0446. be attributed to the combination of single ion detection limits [7] and array detection in tandem four-sector instruments [7-10]. Advances in ionization methods, such as electrospray [11-13] and matrix-assisted laser desorption $[14,15]$, combined with advanced tandem instrumentation, will further the promise of rapid, high sensitivity mass spectrometric sequencing.

Two mass spectrometric approaches are currently used in peptide sequencing. Both are known as tandem mass spectrometry (MS/MS) bul are distinctly different in the mass analyzers employed and the modes of fragmentation typically observed. "Low energy collision-induced dissociation (CID)" is typically practiced on triple quadrupole or hybrid mass spectrometers and results in a smaller set of observed sequence ions compared with "high energy CID," which generally requires sector based instrumentation. (Detailed comparisons have been reported recently $[16,17]$.) Computer-assisted strategies for analyzing low energy CID spectra of peptides have been reported [18].

Modern tandem double focusing mass spectrometers use the first mass spectrometer to select an iso- 
topically pure molecular ion, a gas cell to collisionally energize the incident ions, the second mass spectrometer to mass analyze the resulting fragments, and an electro-optically coupled microchannel plate array for detection within a pseudofocal plane that may cover a $4 \%$ to $-20 \%$ mass range at nominal mass resolution $[9,19]$. By using this technology tandem spectra can now be obtained in minutes on picomole amounts of peptides from mixtures such as enzymatic proteolysis fragments.

The task of protein sequence analysis is to determine the ordered series of amino acids that comprise a peptide chain. Certain characteristics of peptides, relative to other classes of organic molecules, simplify their structural characterization by mass spectrometry. Genetically encoded peptides and proteins are linear, and amino acids linked by peptide bonds maintain a consistent $\mathrm{N}$ - to $\mathrm{C}$-terminal orientation. The genetically encoded alphabet consists of only twenty residues, twa pairs of which are isobaric ${ }^{1}$. While these factors aid in structural identification, sequencing remains a challenge because there are $20^{n}$ sequences of peptides $n$ residues long. This translates to greater than $2 \times 10^{7}$ possible sequences of nominal mass 800 u [20]. Consideration of modified amino acids increases complexity and counters the advantages conferred by genetically encoded compositions. Still, identification of modified amino acids remains a major strength of mass spectral techniques [6].

Mass spectrometry has several advantages over conventional sequencing: the ability to analyze rare or modified amino acids, superior performance on mixtures, and speed of analysis. Automated Edman degradation, the most widespread method of acquiring sequence data, typically utilizes chromatographic methods to separate and identify the amino acids derived from the peptide or protein being degraded. Identification of each residue is based on comparison to the retention time of a standard, and explicit preparation for nonstandard residues is required [21]. In contrast, mass spectrometry does not require advance preparation for the identification of novel residues prior to data collection [22]. One important class of modifications to peptides and proteins is $\mathrm{N}$-terminal blocking groups that prevent direct Edman sequencing but cause little problem to mass spectrometric methods beyond consideration during spectral interpretation. Mixtures are extremely difficult and often. impossible to deconvolute in chemical sequencing experiments, whereas MS/MS employs an initial gasphase separation based on mass-to-charge ratios that eliminates the need for complete purification in most cases. Edman degradation takes $30 \mathrm{~min}$ to $1 \mathrm{~h}$ per

\footnotetext{
${ }^{1}$ Two amino acids, leucine and isoleucine, are isomeric but may be distinguished by side chain fragmentations that are frequently observed under high collision energy regimes [28-30]. The remaining isobaric pair, lysine and glutamine, tequires further information but often inferences may be made based on sample origin and the expected effects of the basic amino group of lysine on observable fragment ions. Definitive results are obtainable by acetylation.
}

cycle [23], while acquisition of a complete CID spectrum of a peptide of $M_{r} \sim 1500(-12-16$ residues) takes less than $5 \mathrm{~min}$. Unfortunately, at this stage interpretation of peptide fragment ion spectra can require hours to days of an expert's time.

Interpretation of a mass spectrum is accomplished either by (1) correlation of observed fragments with those predicted from a postulated sequence and empirically derived fragmentation rules, or by (2) building sequences from among the possible interpretations of the fragments observed. The difference between these two approaches is that the latter uses mass spectral data to generate sequence, whereas the former merely tests a series of postulated sequences and their predicted spectra for fit to data. An important variant of correlation methods is the partial correlation method $[20,24]$ in which spectra are predicted based on a limited guess (such as a C-terminal amino acid) and evaluated for fit to data. If a partial sequence guess passes a discrimination step, it then becomes a "seed" for subsequent extension by one or two amino acids. This process is repeated until a sequence guess meets (is reported) or exceeds (is discarded) the mass of the protonated peptide. The occurrence of an unexpected residue (not part of the guessing algorithm) precludes successful interpretation by such an algorithm. Inclusion of additional residue masses increases analysis time.

The second approach, building sequences directly from the data, is the one we have undertaken and is the subject of this article. Our method's principal advantages are its potential to discover unanticipated answers and its reliance on the CID spectra themselves as the initiators of sequence assignment. It should also have advantages in speed and in the ability to yield partial sequences from spectra even when complete sequencing may not be possible, either because the present algorithm is not optimal or because the necessary information is not contained in the spectrum. One difficulty with both kinds of correlation schemes arises from inexperience with the patterns found within CID spectra. It is currently impossible to predict confidently the complete high energy CID spectrum expected from a given amino acid sequence, making correlation of predicted sequences problematic. We therefore propose to develop and implement not only algorithms to extract sequence information, but also tools that will eventually generate rules describing the expected observable sequence ions, maximizing our ability to discriminate between candidate sequences. An ultimate goal of this work is to provide accurate data reduction in real time so as to fully complement mass spectrometry's inherent speed of analysis.

\section{Method}

Our MS/MS instrumentation (Kratos Concept IHH, Manchester, UK) is of four-sector EBEB geometry with 
a gas collision cell between the two double focusing mass spectrometers, as described in detail elsewhere [10]. Peptides are dissolved in a liquid matrix and sputtered with a $\mathrm{Cs}^{+}$ion beam. The most commonly used matrices are glycerol/thioglycerol $(1: 1$ or $2: 1$, with or without acidification), nitrobenzyl alcohol, and dithiothreitol:dithioerythritol mixture $(4: 1)$. The first double focusing mass spectrometer selects and transmits a single isotopic species into the collision cell. Fragment ions are produced by CID with He in the gas cell. The collision cell potential can be varied, but for most of these experiments it was set to give a collision energy of $4 \mathrm{keV}$. Fragments from singly charged parents are mass analyzed by MS-II and detected on an electro-optically coupled microchannel array that simultaneously detects masses over a $4 \%$ range with a typical resolution of 1500 . Channel/array data are saved on computer (Eclipse S280) and transferred via ethernet to a Sun $3 / 60$ (Sun Microsystems, Mountain View, CA) for analysis and storage on hard disk.

Programs have all been written in the $C$ programming language using the standard compiler supplied with Sun Unix version 4.01. Initial programs were also developed in $C$ on a Macintosh computer using Think $C$ version 4.0 (Symantec, Cupertino, CA). An interactive graphical learning tool that helps a novice visualize sequence choices and utilizes a color Macintosh display will be described elsewhere.

Peptides employed in this study were most often obtained from specific enzymatic proteolysis of proteins, followed by partial purification by reverse phase high-performance liquid chromatography [25]. Other sources include isolates of bioactive peptides from amphibian skin secretions [B. Gibson, unpublished datal, synthetic peptides, and chemically derivatized peptides of various origins. The peptide spectra analyzed represent the efforts of many investigators working within the UCSF Mass Spectrometry Facility who have generously allowed the use of their unpublished data.

A peak detection routine was written specifically for this work to generate a reliable set of peak mass and peak height values from raw data. Our peak detection scheme seeks local maxima with "reasonable" peak shape, as defined by the local slope one channel each to the left and right of the pcak top as a percent of peak height. It alerts the operator to questionable peak shapes or masses inconsistent with usual peptide accurate masses. This routine saves the $n$ most abundant peaks, where $n$ is either user-specified or $10 \%$ of the precursor mass (default). The number of peaks is intentionally chosen large to include many seemingly insignificant peaks. Mass and peak height values are recorded for these peaks and stored as an ASCII file. Peak height is reported in millivolts, the same scaling presented in the Mach3 (Kratos) raw data package. Actual sequencing algorithms use this list of ion masses and peak heights, with one impor- tant modification, to produce files with complete and partial interpretations of spectra. All masses are initially recorded with $0.01 \mathrm{u}$ precision and typically better than \pm 0.2 u accuracy, but are converted to nominal mass within the sequencing algorithm. The conversion applies a correction factor of 0.9995 (the reciprocal of the typical peptide mass excess) and then rounds to the nearest integer. Thus, 1000.49 becomes 1000 and 2001.13 becomes 2000 . Conversion to nominal mass speeds and simplifies further comparison between masses. This conversion does not lose significant information as peptide mass excess (as a percent of peptide mass) shows little variation. The table of peaks can be edited manually to correct for improperly calibrated data, separately from the sequencing program, although this has seldom proved necessary.

\section{Sequencing Algorithm}

Fragment ions from peptide molecular ions have been characterized and a suitable nomenclature has been established [26]. More than twenty [27] different ion types have been described thus far for high energy collisions and a subset of these is also present in low energy CID spectra [16]. Most of these ions are of potential use in strategies designed to turn peptide tandem mass spectra into amino acid sequences. The types of ions used in our algorithm are the six peptide backbone cleavage products $(\mathbf{a}, \mathbf{b}, \mathbf{c}, \mathbf{x}, \mathbf{y}$, and $\mathbf{z}+\mathbf{1})$ and three resulting from important side chain losses (d, $v$, and $w$ ) [27-30]. The single amino acid immonium inns are also used to restrict the amino acid compositions considered by classifying each amino acid as allowed, required, or excluded [31].

Table 1 lists the ions used in our algorithm along with formulas for calculating their masses. These formulas may be rewritten to describe all ions based on a single sequence-ion type. In our case we have chosen the $y$ type ion as shown in Figure 1. (Two values of $\mathbf{w}$ ions are currently matched with $\mathrm{R}_{a}$ as $\mathrm{H}$ and $\mathrm{CH}_{3}$.) This pattern is used to find sets of potentially related

Table 1. Principal ions used in the algorithm with formulae for calculating ion masses based on known sequence

\begin{tabular}{|c|c|c|}
\hline Type & Simple structure & Generic formula \\
\hline $\mathbf{a}_{1}$ & $+\left[\mathrm{NH}_{2}=\mathrm{CH}[\mathrm{R}]\right]$ & $\Sigma a_{i}-27$ \\
\hline $\mathbf{b}_{1}$ & {$\left[\mathrm{NH}_{2} \mathrm{CH}(\mathrm{R}) \mathrm{CO}\right]^{+}$} & $\Sigma a a_{i}+1$ \\
\hline $\mathbf{c}_{1}$ & ${ }^{+} \mathrm{H}\left(\mathrm{NH}_{2} \mathrm{CH}(\mathrm{R}) \mathrm{CONH} \mathrm{N}_{2}\right]$ & $\Sigma a_{i}+18$ \\
\hline$d_{2}$ & ${ }^{+}\left[\mathrm{NH}_{2} \mathrm{CH}(\mathrm{R}) \mathrm{CONHCHR}, \mathrm{I}\right.$ & $\mathrm{Eaa}_{\mathrm{i}}+43+\mathrm{R}_{\mathrm{a}}$ \\
\hline $\mathbf{v}_{2}$ & ${ }^{+}\left[\mathrm{NH}_{2} \mathrm{CHCONHCH}(\mathrm{R}) \mathrm{COOH}\right]$ & $\Sigma a_{i}+74$ \\
\hline $\mathbf{w}_{2}$ & ${ }^{+}\left[\mathrm{R}_{\mathrm{a}} \mathrm{HC}=\mathrm{CHNHCH}(\mathrm{R}) \mathrm{COOH}\right]$ & $\Sigma a a_{i}+72+R_{a}$ \\
\hline$x_{1}$ & ${ }^{+}[\mathrm{CONHCH}(\mathrm{R}) \mathrm{COOH}]$ & $\operatorname{saa}_{1}+47$ \\
\hline $\mathbf{y}_{1}$ & ${ }^{+}\left[\mathrm{NH}_{3} \mathrm{CH}(\mathrm{R}) \mathrm{COOH}\right]$ & $\Sigma \mathbf{a a}_{1}+19$ \\
\hline $\mathbf{Y}_{1}-2$ & ${ }^{+} \mathrm{H}[\mathrm{NH}=\mathrm{C}(\mathrm{R}) \mathrm{COOH}]$ & $\Sigma a_{i}+17$ \\
\hline$z_{1}+1$ & ${ }^{+} \mathrm{H}[\mathrm{CH}(\mathrm{R}) \mathrm{COOH}]$ & $\Sigma a a_{1}+2$ \\
\hline
\end{tabular}

Substituents $F_{2}$ on $d$ and $w$ ions are normally $H$ for amino acids that are not branched at the beta carbon. Other possibilities are $\mathrm{CH}_{3}$ for $\mathrm{Val}$, Thr, and lle, as well as $\mathrm{OH}$ for Thr and $\mathrm{C}_{2} \mathrm{H}_{5}$ for lle. 

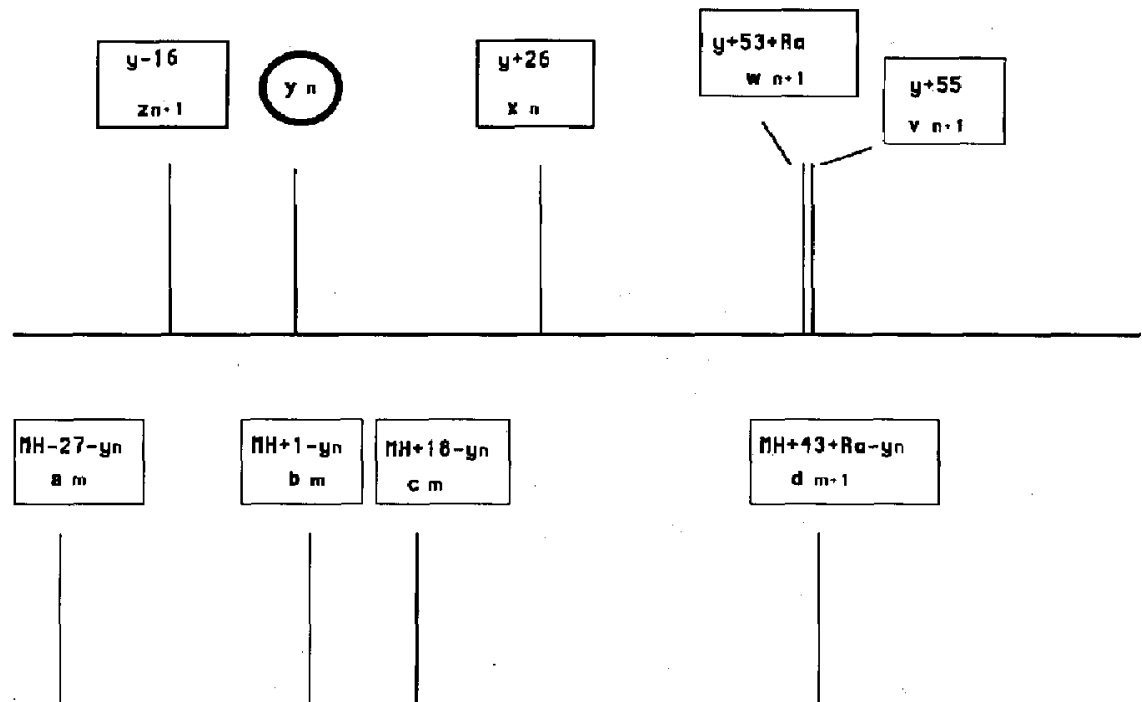

\begin{abstract}
Figure 1. The set of ions that comprises an ion family. Algebraic relationships between individual sequence ions are shown. $R_{a}$ is taken as $H$ for this figure. Currently, our algorithm also allows for $R_{a}$ as $\mathrm{CH}_{3}$ for $w$ ions but distinguishes this ion in $y$-center and link reports by using uppercase $(w)$. It should be further noted that the nomenclature used in our program differs from that proposed by Biemann and co-workers [28], labeling $z_{n}+1$ simply as $z$. This is in keeping with the goal of simplifying the nomenclature to refer to the most commonly observed ion, as has been done with $y$ and $c$ ions. For this article we have used the $\mathbf{z}_{n}+1$ nomenclature except in Figure 4 , where the +1 has been omitted to alleviate clutter.
\end{abstract}

ions. These relationships are independent of specific amino acid sequence and instead derive from the repetitive polymeric nature of the peptides. The pattern matching approach capitalizes on the redundant sequence information present in high energy CID spectra to reinforce a common signal and is the cornerstone of our algorithm.

There are three distinct steps in our sequencing routine: transformation, linking, and sequence tracing. The transformation step begins with the mass of the most abundant ion in our analysis set. This mass is used as a "seed ion" to generate an ion matrix like that shown in Table 2. The matrix is generated by postulating the seed ion as each ion type and calculating the related ions" masses ${ }^{2}$ from the formulas presented in Figure 1. Each "ion family" (an ion family is a row within the matrix) is then scored by the number of experimental matches and the sum of their corresponding peak heights. Two arrays with dimensions consistent with the size of $\mathrm{MH}^{+}$are used to record primary and transformed data and these enable direct checking (look-up table) for the presence of any given real or transformed mass. Ion families

\footnotetext{
${ }^{2}$ We refer to fragment ion masses, rather than mass-to-charge ratios, in this article because the fragment ions are known to be singly charged. A multiply charged ion formed in the (flaated) collision cell of a four-sector tandem machine would not appear at all in the fragment ion spectrum because it would not be simultaneously transmitted through the electric and magnetic sectors of MS-II during the linked scan [32] of these two fields.
}

whose scores exceed a set threshold are recorded in the second array and indexed according to the mass of the $y$ ion belonging to this family. Each $y$-centered family record contains the count of members, the sum of their peak heights, and a list of the types of ions found (an actual $y$ ion does not need to be found in

Table 2. The ion matrix shown depicts the transformation of the "seed" mass of $468 \mathrm{u}$ (in bold) from a pepide with a protonated molecular ion of $1093 \mathrm{u}$

\begin{tabular}{l|l|l|l|l|l|l|l|l}
$\mathrm{a}$ & $\mathrm{b}$ & $\mathrm{c}$ & $\mathrm{d}$ & $\mathrm{v}$ & $\mathrm{w}$ & $\mathrm{x}$ & $\mathrm{y}$ & $\mathrm{z}$ \\
\hline $\mathbf{4 6 8}$ & 496 & 513 & 539 & 653 & 652 & 624 & 598 & 582 \\
\hline 440 & 468 & 485 & 511 & 681 & 680 & 652 & 626 & 610 \\
\hline 423 & 451 & 468 & 494 & 698 & 697 & 669 & 643 & 627 \\
\hline 397 & 425 & 442 & 468 & 724 & 723 & 695 & 669 & 653 \\
\hline 653 & 681 & 698 & 724 & 468 & 467 & 439 & 413 & $\frac{397}{}$ \\
\hline 652 & 680 & 697 & 723 & 469 & 468 & 440 & 414 & 398 \\
\hline 624 & 652 & 669 & 695 & 497 & 496 & 468 & 442 & 426 \\
\hline 598 & 626 & 643 & 669 & 523 & 522 & 494 & 468 & 452 \\
\hline 582 & 610 & 627 & 653 & 539 & 538 & 510 & 484 & 468
\end{tabular}

Rows within the ion matrix represent "ion families" as defined in the text. Ions types are listed at the top of each column. The symmetrical relationships of $\mathrm{N}$ - and $\mathrm{C}$-terminal ion pairs should be moted. Figure 2 shows the matches found for ion families that include the peak at $468 \mathrm{u}$ in the peptide spectrum from which this example was derived. 
the data to establish a "y-center"). This transformation continues for each new seed ion whose peak height remains above a cutoff that is either at the fiftieth percentile (default) or a value specified by the user. Duplication of effort is eliminated in the transformation step, by using the $\mathbf{y}$ mass of each family to test if that family has already been found (using another more abundant family member as the seed ion). A strategy has been described by Bartels [33] utilizing a similar transformation into what was termed a "sequence spectrum" but few details of actual sequence generation were provided.

After our transformation has been completed, $y$ centers are then linked. A link is generated when two $y$-centers differ by the residue mass of an amino acid. Links are scored by the peak height and number of ions in each of the linked $y$-centers as well as by the similarity of ions observed. This last score is termed the "common ion count" and is the number of same type ions found in both sets. For example, two $y$ centers, $m / z 650=\mathbf{a}, \mathbf{b}, \mathbf{y}, \mathbf{z}+\mathbf{1}$ and $765=\mathbf{v}, \mathbf{w}, \mathbf{y}$, $z+1$, have common ion count $=2$ for the $y$ and $\mathbf{z}+\mathbf{1}$ ions in common and they are linked by Asp whose residue mass is 115 . Transformation to " $y$-ion space" could also allow for calculation of the mass of unanticipated or modified amino acids. This requires recognition of two $y$-centers that are strongly supported yet yield poor sequence interpretations (i.e., have poor or missing links) through this mass region. In such a case the mass of the new amino acid is simply the difference between the two strongly: supported $y$-center masses. Current scoring protocols are poor, however, at recognizing such a link. At present, manual analysis of transformed data is the best way of locating a novel amino acid, in our experience.

Once the $y$-centers have been linked, sequences are generated by tracing forward from low to high $y$-mass. Starting points can be restricted by knowledge of enzymatic conditions tused in generating peptides. Tryptic peptides would most commonly be restricted to an Arg or Lys at the C-terminus, though this restriction would likely prevent successful interpretation of a protein's C-terminal peptide as well as any peptides derived from nonspecific cleavage. If not restricted by the user, sequences may begin with any of eighteen choices: the sixteen nonisobaric amino acids or one member of the isobaric pairs of Leu/lle and Lys/Gln. The algorithm presently uses the single

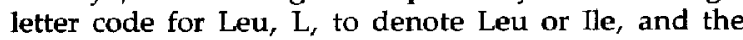
single letter code for Lys, K, to denote Lys or Gln, and makes no attempt to differentiate within these pairs (see footnote 1). Modified $\mathrm{N}$ - or C-terminal groups are allowed but must be indicated by the user to be considered in the final sequences. Sequence tracing consists of recursively moving up in sequence by the predetermined links. Sequences are complete if they link to $\mathrm{MH}^{+}$(the final $y$-center). Completed sequences are rescored to include immonium and other low mass amino acid specific ions and to ex- clude ions that fit a generic $y$-center but do not fit the specific sequence. An example of this would be the exclusion of a $w$ ion from the lower side of a Gly link because glycine has no side chain. Scores of completed sequences include the count of sequence ions found, the sum of peak heights, and the sum of the common ion counts from the link scores. A further type of score is detailed in the Discussion.

False leads are reduced by a back tracing routine that was added to prevent sequence tracing along paths that cannot be linked to $\mathrm{MH}^{+}$. This is accomplished by first flagging those $y$-centers that are linked to $\mathrm{MH}^{+}$by a single residue mass. Then in a single pass though the array of $y$-centers beginning at the high mass end and progressing downwards, the down links of each "flagged" $y$-center are flagged. This procedure will flag any $y$-center that can be completed but does not need to explore all the pathways by which completion can occur to do this. The tracing algorithm can then disregard links to $y$-centers that have not been "flagged" as being able to be completed. This can have a large effect in reducing the time needed to complete the sequence tracing step.

Completed sequences are written to an ASCII file, as are lists of $y$-centers and $y$-center linkage data. These files then serve to assist manual evaluation of the spectrum.

\section{Results}

Examples follow for the three major steps of our algorithm. The first example depicts the creation of $y$-centers. Figure 2 shows the various $y$-centers derived from the seed mass of $468 u$ which is actually a $b$ type ion. The true fit is quickly recognized as the most likely based on the types of ions often found together in an ion family. Any pair of $\mathrm{N}$ - and C-terminal ions can trade identities in support of an alternative $y$ center. Two of the alternative ion families illustrate this symmetry-based problem where a C-terminal ion (in this example the $y$ and $z+1$ ions) from the correct set trades its context with the $b$ ion at $468 u$. The 468 $\mathbf{u}=\mathbf{a}$ and $539 \mathrm{u}=\mathbf{d}$ fit results from the coincidence of $a$ and $d$ ions differing by the residue mass of the amino acid $(\mathrm{Ala}=71)$ that separates these two $b$ ions. The $468 \mathrm{u}=\mathrm{w}$ and $\mathbf{4 4 0} \mathrm{u}=\mathrm{x}$ match arises from the coincidence of $28 \mathrm{u}$ separating this pair and $a, b$ pairs. The final false signal of $468 \mathrm{u}=\mathbf{d}$ and $695 \mathrm{u}=\mathrm{x}$ is simple coincidence. In this example, six of nine possible $y$-centers survive the discrimination step for families derived from $468 \mathrm{u}$. Rules to exclude some sets, such as the seldom observed $d, x$ pair, have not yet been incorporated into our algorithm. Distinguishing between real and artifactual or coincidental $y$-centers is not trivial. Scoring options will be discussed below.

Different $y$-centers are linked together when they differ in their $y$-masses by the residue mass of an amino acid. In Figure 3 the $y$-center $602 \mathrm{u}$ is correctly linked up to $715 \mathrm{u}$ by a leucine/isoleucine and linked 


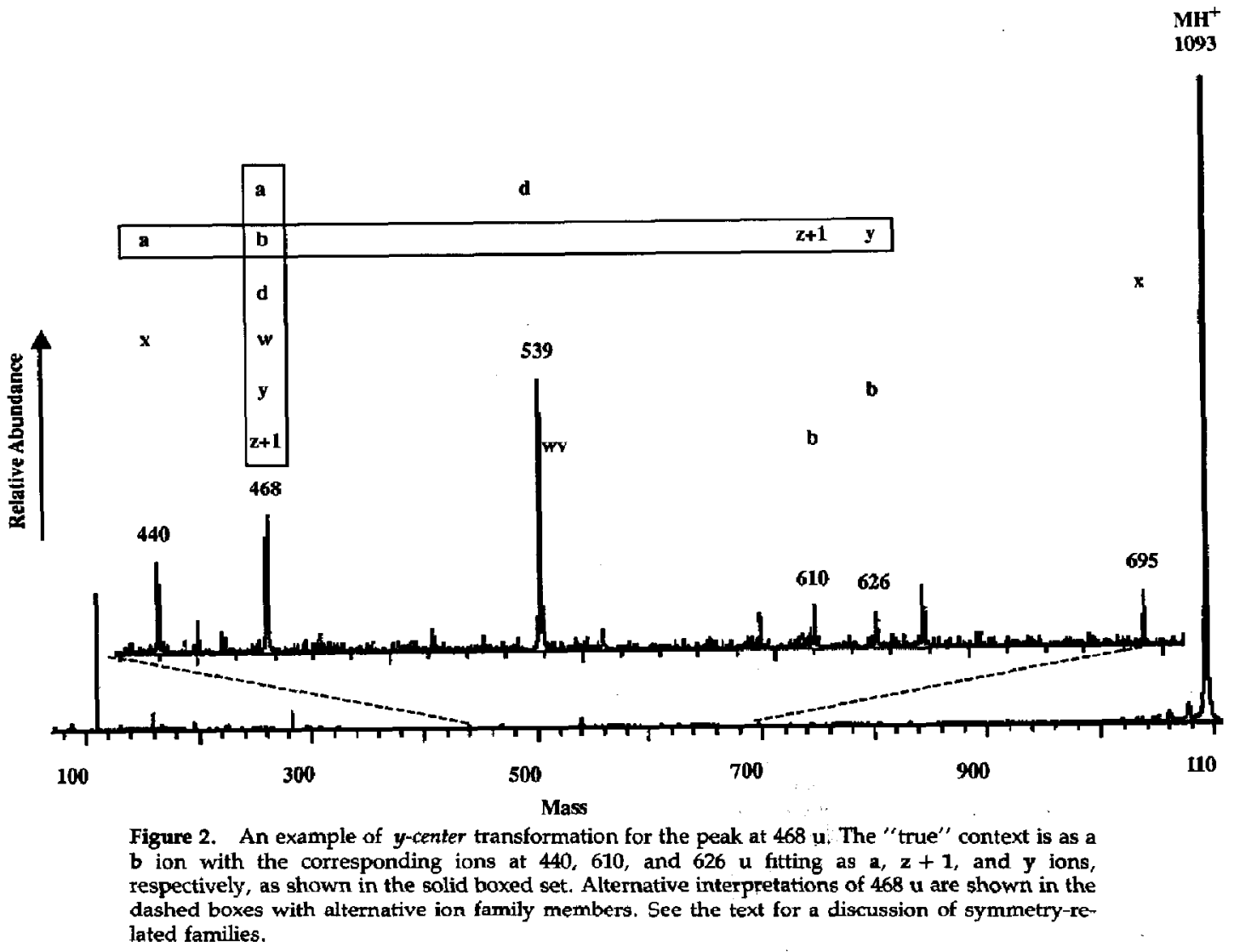

down to $503 \mathrm{u}$ by a valine. Correct links may sometimes be deduced by similarity of ion content, as in this example. In many other instances, discrimination is not possible prior to consideration in the context of a completed sequence. This results in carrying many incorrect links.

Correct sequences are generally accompanied by similar sequences with inversions and dipeptide substitutions. A peptide of $\mathrm{M}_{\mathrm{r}} 901$ gave the top four scoring sequences as SLDFYTR(46840), LSDFYTR(45978), SLTYFDR(44490), and LSTYFDR(43628). The scores (in parentheses) are the sum of the peak heights of the sequence and immonium ions for the given sequences as reported by the program. In this example, the $\mathrm{N}$-terminal pair inverts between the two best scoring candidates, which have similar scores. Residues 3-6 also invert in a less typical fashion. The order of the $\mathrm{N}$-terminal pair is often difficult to determine due to the presence of immonium ions and internal acyl or immonium fragments. Careful examination of the spectrum might have enabled a best guess from among these possibilities based on the presence or absence of internal fragments while the small differences between the peak height sum scores alone would have been inadequate to confidently se- lect from among these candidates. From the same tryptic digest, however, the previously run peptide gave the top scoring sequences of KSLDFYTR(11603), KSDLFYTR(11252), KLSDFYTR(11250), and SLKDFYTR(11132). By using this larger peptide in combination with the smaller it was possible to confidently assign the sequence KSLDFYTR. This spectrum is shown in Figure 4. The lysine on the $\mathrm{N}$-terminus was chosen over the isobaric amino acid glutamine (by us, not by the algorithm, which does not attempt that distinction). In this example the choice derives from comparison of these two peptides and the fact that trypsin was used to digest the protein, indicating that the larger peptide was the result of incomplete enzymatic digestion. It should be noted that in the absence of the spectrum of the shorter peptide in this digest, one would have been tempted to reverse the argument and call the lysine a glutamine because lysine would then represent a partial (unlikely?) digestion product.

\section{Discussion}

In mass spectral sequencing, individual amino acids are most simply assigned from successive sequence 


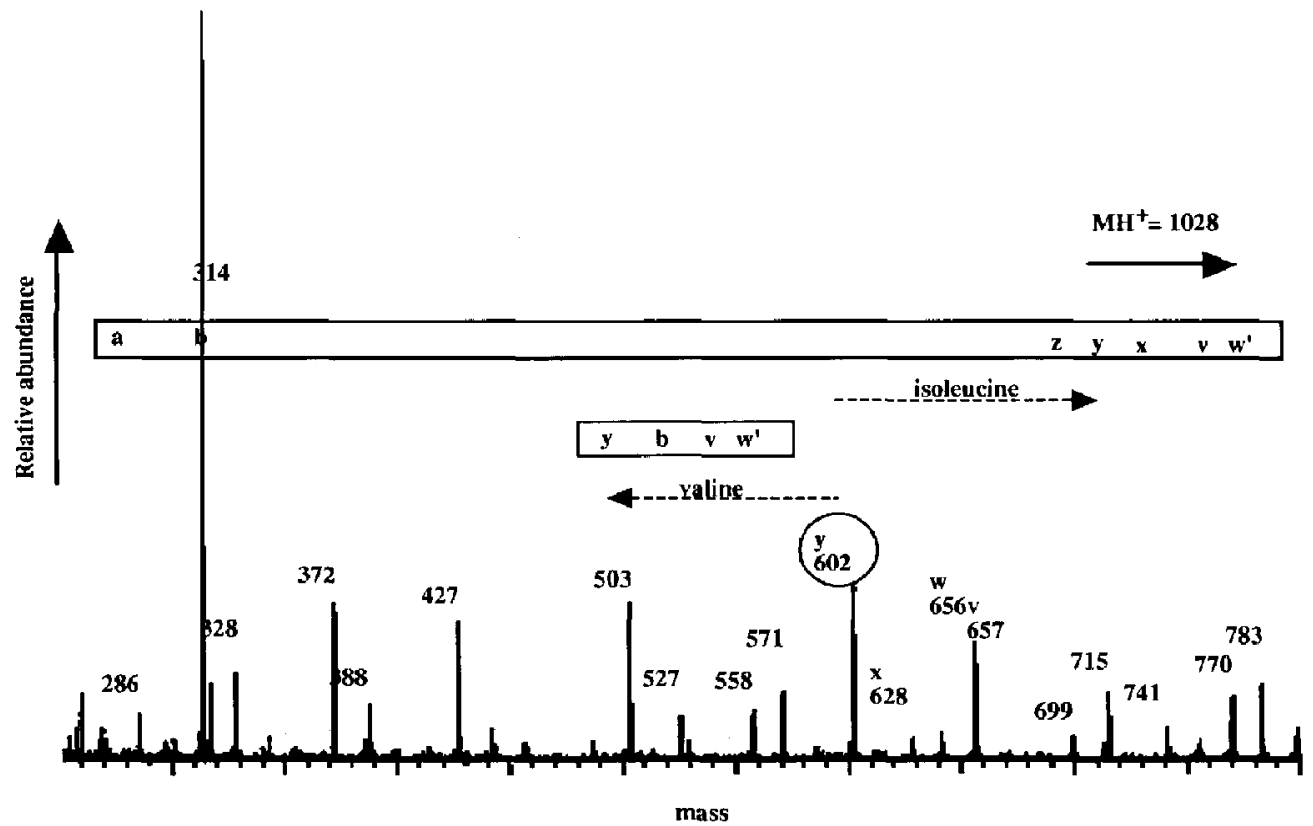

Figure 3. Amino acid links between $y$-centers are detected by comparing $y$-center mass differences with known amino acid residue masses. Correct, as opposed to artifactual, links are often distinguished by similar types of sequence ions. In the example the ion family with $y=602$ $\mathbf{u}$, also includes $\mathbf{b}, \mathbf{x}, \mathbf{w}$, and $\mathbf{v}$ ions as labeled immediately above the corresponding peaks. The best up and best down link $y$-families are shown and boxed. Ions labeled $w^{\prime}$ represent $\mathrm{R}_{\mathrm{a}}=\mathrm{CH}_{3}$ (refer to Figure 1 for explanation of $R_{a}$ ).

ions of the same type. For the peptide Gly-Ala-Asp. the difference between $a_{1}$ ion at $m / z 30$ and $a_{2}$ at $m / z 101$ is $71 \mathrm{u}$ and corresponds to Ala at position 2 . The same difference of 71 is found between $\mathbf{b}_{1}$ $(m / z 58)$ and $b_{2}(m / z 129)$. In both cases the difference is the addition of $\mathrm{C}_{3} \mathrm{H}_{5} \mathrm{NO}$. Information about Ala $a_{2}$ could also be derived from $b_{1}$ and $a_{2}$ if we knew that $m / z 58$ was $\mathbf{a} b$ ion and $m / z 101$ was an $\mathbf{a}$ ion. Of course, the difficulty is that we do not experimentally measure $b$ or a character, we only measure the fragment ion masses (see footnote 2). Any strategy designed to deduce sequence from spectra must, in fact, label the individual ions, either by mathematical pattern recognition algorithms as a prelude to sequence assignment or, as is more common, by comparison with masses calculated from a proposed sequence. Our choice has been to attempt the pattern recognition approach as a more direct path from data to sequence and one that may eventually allow for the automatic recognition of novel amino acids. In this pattern-based approach, identified ions are transformed to a consistent context from which sequencebased relationships between ions may be deduced.

The pattern we use is that determined by the peptide nature of the sample, that is, the linear, polymeric repeat of amino acids. The algorithm separates the sequence-independent step of $y$-center de- tection from consideration of sequence-dependent relationships, the understanding of which is still evolving. Instead, it takes maximal advantage of what is known about the sample (that it is a polymer of alpha amino acids) before addressing the question of sequence.

The linking process compares existing y families for linking rather than guessing $y$ family extensions, and therefore can allow for more than the standard genetically encoded amino acids without slowing the linking process. This is because the linking step requires, at most, a single comparison between each pair of $y$-centers, not a loop through all extensions for each $y$-center. Each comparison results in a mass difference that is used directly in a look-up table. Look-up tables are used throughout our algorithm to realize significant speed advantages. Instead of using a loop to search a list of ions for the presence of a given mass, a look up table allows onc to directly inquire at that mass to determine whether it is present. This is accomplished both for real masses and for $y$-centers by using large arrays whose $n$th element is either zero, to indicate the absence of a mass $n$, or contains the address in memory of the data structure (pointer) that contains further information on that mass. In the case of linking, the look-up table holds the identities of residues with a particular mass. Links 


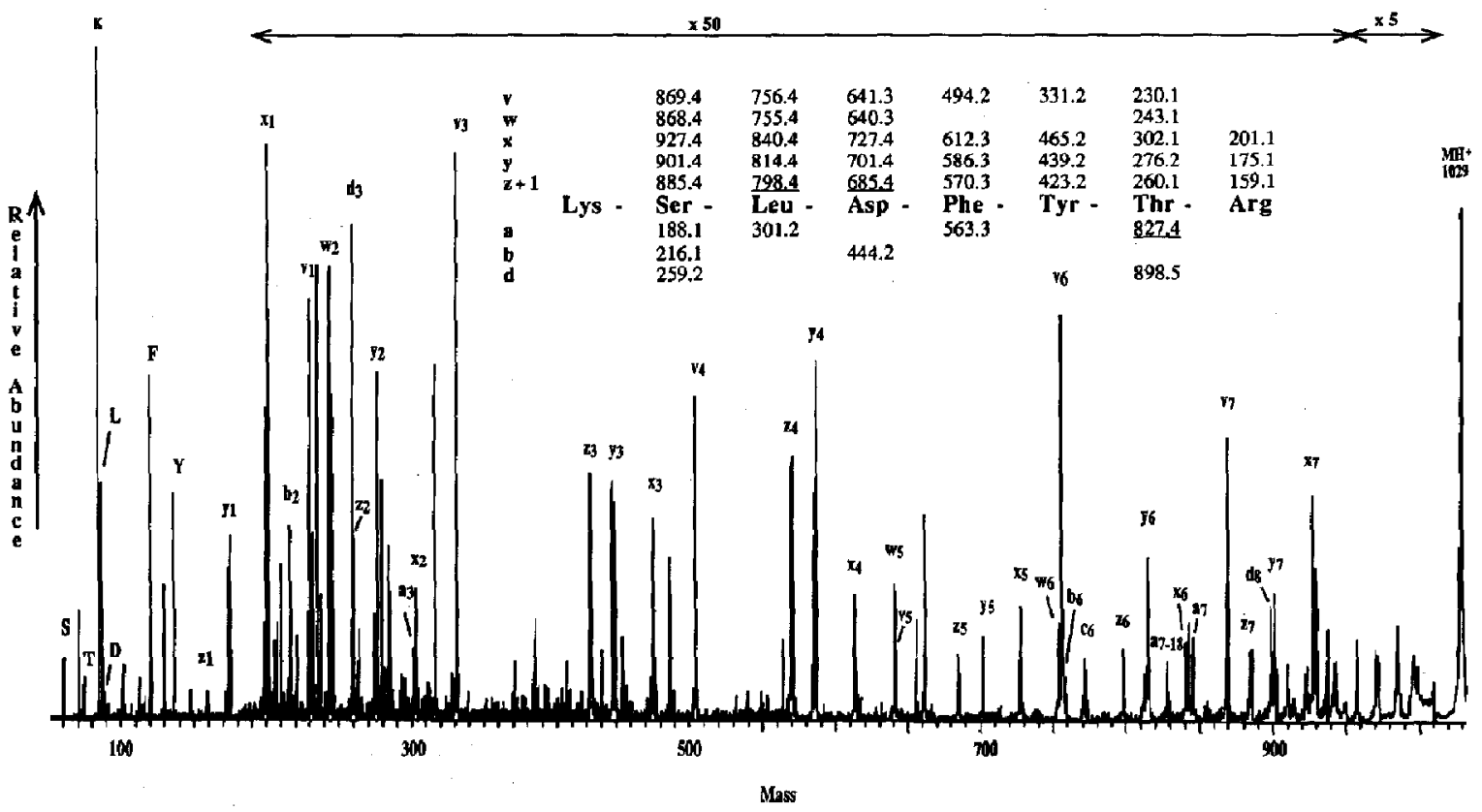

Figure 4. A spectrum with completed sequence interpretation. In this example, the sets of $z+1$, $y$, and $\mathbf{x}$ are quickly recognized. The dominance of $\mathrm{C}$-terminal ions is readily accounted for by the presence of an Arg residue as the C-terminal amino acid. Currently unassigned, yet relatively abundant, are the ions at $316,477,655$, and $661 \mathrm{u}$. The peak at $70 \mathrm{u}$ could be assigned to Lys or AIg and so was not labeled. The peak at $129 u$ could be $b_{1}$ or the immonium ion for Arg and was also not labeled. Other amino acid specific low mass ions are labeled by uppercase single letter code. Ions denoted $z_{n}$ are in fact $z_{n}+1$ ions according to the nomenclature presented in Table 1 .

are recorded in both the higher and lower mass $y$ centers in down and up links, respectively. This allows sequence tracing in both directions.

Restrictions on amino acid composition are often possible from a survey of the low mass portion of a peptide's CID spectrum. Amino acids that normally produce strong characteristic immonium ions are disallowed if their expected immonium ions are not present. Similarly, the presence of characteristic ions can be used to require their presence in completed sequences. Disallowed residues are excluded in the linking steps, while required residues are used as a filter on completed sequences. Because the program is fast, requiring between 10 and $60 \mathrm{~s}$ depending on the number of sequences reported, reanalysis of a spectrum without restrictions is quickly accomplished and is recommended to test the robustness of the best scoring sequence solutions. Additionally, restrictions can be used to reduce the number of sequences found. This can be enlightening even when an excluded amino acid is in the sequence of the peptide that is being analyzed! This seemingly strange result arises due to our ability to compare rapidly the best scoring sequences with and without restriction.

The current version of our algorithm places two levels of restrictions on the number of completed sequences that are reported. The first restriction begins with the 101st sequence found and requires it to score better than $75 \%$ of the best current score. The second restriction is termination of sequence tracing after 400 sequences have been reported. These arbitrarily chosen restrictions are in place to focus the user back to the parameters usable to limit the number of sequences generated. The presence of such a large number of sequences indicates that too many $y$-centers are passing the initial discrimination step. One means of reducing the number of proposed sequences is to increase the number of $y$-centers that are rejected. The two parameters usable for this are the number of family members that must be found and the minimum for their summed peak heights. A further way to decrease the number of $y$-centers is to reduce the number of ions used as seeds to generate the ion matrix. This in effect requires at least one of the $y$-center's members to be above the peak height threshold for matrix generation. Another option is to use strong signals from within the originally proposed sequences to restrict the composition of reported sequences. In this case the restrictions are not based on immonium ions but on strongly supported partial sequence(s) that may have been detected in the initial sequencing attempt. 
Scoring can serve two goals in sequence analysis: to provide both relative and absolute rankings. A CID spectrum has two obvious dimensions: mass and ion abundance. Masses are used to identify individual peaks, and abundances are often used as a measure of importance in determining a sequence. Boolean descriptions are simplest: a peak at a given mass is either present or not, but this logic requires imposition of an arbitrary abundance threshold below which signals do not count. Simple summation of ion abundances seems to address this difficulty by weighting an ion's presence by its abundance and therefore reduces thresholding effects, but directly correlating ion significance with abundance is dubious. There does not exist a compelling reason to assert that an ion that is ten times as abundant is ten times more meaningful or valuable for ranking alternative interpretations of data. Still, counts and abundance sums present information that remains free from bias potentially imposed by an interpretation scheme. In the method described by Johnson and Biemann [24], the principal score assigned to a sequence is the sum of the abundances of identified ions normalized to total ion abundance. This ratio does describe the degree to which a spectrum has been fully interpreted, yet fails to weigh the degree of satisfaction applied to individual ion assignments or to the completed pattern of sequence and nonsequence ions. A more complex scoring scheme was used to evaluate sequence extensions during their sequencing algorithm. The algorithm, described above, propagates partial sequences by one amino acid and evaluates the quality of fit to data. Extensions bear some similarity to our links. Extension scores are broken down into $\mathrm{N}$-terminal and C-terminal scores that selectively exclude certain ion types if others are not found. For example, an Asn extension might yield a $d$ ion but no score would be given it if the corresponding a ion was not also found. Extension scores are further modified based on prior subsequence, including potentially discounting extension scores by $50 \%$ or $80 \%$ depending on the presence or absence of basic amino acids. The rationale given for this scoring scheme is that it is based on previous experience with CID spectra of known peptide scquences. Difficulties arise in the use of arbitrary constants to modify scores and from the lack of rigor in evaluating the effects of these arbitrarily selected modifiers. Despite these concerns we currently lack a significantly better method for scoring sequence fits to CID data.

We use a variety of scores to describe $y$-centers, links, and completed sequences. While attempts have been made to generate overall scores for ranking sequences, the current emphasis is on simple descriptions to aid manual analysis of results files. Scores for $y$-centers are the number of family members found, the sum of their peak heights, and a list of ion types found. Link scores allow for the context applied by the link, revising the list of ions to those pertinent to the link. For example, an uplink by the residue mass of glycine would not include $\mathbf{v}$ or $\mathbf{w}$ ions in the lower mass $y$-center or a $\mathbf{d}$ ion in the higher $y$-center. Contextually correct ions are combined into a total count in both directions and the common ion count is calculated. A last ad hoc link score is the product of the summed peak heights of both of a link's $y$-centers and the square of the common ion count. The intent of this score is to emphasize links between similar sets of ions and it has proven useful in distinguishing between otherwise similar scoring sequences. Another scoring scheme for emphasizing continuous series of same-type ions has been reported by Zidarov et al. [34]. The four sequence scores used in our work are the sum of peak heights of identified ions including relevant immonium ions, the count of identified ions, the sum of the common ion count found in the links used in tracing the sequence, and the sum of the ad hoc link scores divided by the length of the peptide.

We envision three major difficulties in producing adequate scores through simple combinations of ion abundances or ion counts. Different amino acids yield somewhat different fragments. Isoleucine and threonine can each produce two types of $d$ ions and two types of $w$ ions while glycine has no $d, v$, or $w$ ions. For a given molecular weight, smaller amino acids give greater chain lengths and so a higher density of possible sequence ions. Lastly, different peptides of the same amino acid composition will not necessarily yield similar types or numbers of sequence ions due to, among other factors, the effects of a fixed remote charge on observable fragment ions. The combination of these factors leads to the conclusion that what might be too few fragments to favor one sequence would be more than expected for another.

An idealized score would need to consider exactly what a postulated sequence should produce in a CID spectrum. It should further compare results for all proposed sequences and produce a confidence index with absolute confidence in a single sequence yielding a score of 1 . Consider two sequences, VDVLRAK and DVVLRAK, identical except for transposition of the frrst two amino acids, having equal likelihood and exclusive of all other possibilities. Scores would then be VDVLRAK $=0.5$; DVVLRAK $=0.5 ;\{D, V\}$ VLRAK $=1$. Creation of such a scoring scheme will require statistical evaluation of a large number of peptide CID spectra. This scheme will most likely require initial proposal of sequences consistent with the data, followed by a second step of predicting what data should result from a given sequence.

\section{Conclusions}

A distinctive difference between chemical (Edman degradation) and mass spectrometric sequencing is the nature of the data. In chemical sequencing, amino acids are removed one per cycle. Identification of each cycle's amino acid derivative is by retention time 
correlation with amino acid derivative standards. Nonstandard amino acids pose problems for that cycle's analysis, yet do not prevent identification of subsequent cycles. Chromatographic constraints in resolving normal standard mixtures preclude extensive preparation for rare amino acid derivatives. Edman sequencing chemistries (gas/liquid/solid phase) also suffer from sample losses that are cumulative and progressive, complicating or preventing analysis of residues near the peptide's C-terminus. Edman sequencing thus has the advantages of cycle-based analysis, resulting in simple interpretation and confidence in partially correct answers. It has the disadvantages of inability to interpret residues for which a standard had not been evaluated, lack of redundancy, which provides consistency checks, and difficulty in completing the analysis of the entire peptide.

In contrast to chemical sequencing, mass spectral sequencing acquires data from the whole peptide simultaneously, providing checks on the completeness of a determined sequence through redundancy and complementarity. This redundancy is at the expense of greater complexity. A high level of redundancy representing a small region of sequence can rob a spectrum of other observable sequence ions and, due to this competition, result in incomplete sequence information. Mass measurement does not require explicit preparation for modified or rare amino acids prior to data collection.

Pattern matching schemes for interpretation of mass spectrometric data have been reported previously [35-38]. While general attempts have failed to meet expectations, some programs have proven quite reliable. The success of most of these programs has been attributed to restriction of the class of compound being analyzed. Peptides certainly represent a special class of compounds in this sense.

The current implementation of our algorithm has proved very good at interpreting certain classes of peptide spectra, especially those obtained from tryptic digests, but has also proved useful with peptides derived from $S$. aureus V8 and other proteases as well as naturally occurring peptide hormones. An excellent example is a tryptic peptide derived from a test peptide used to compare sequencing techriques [39]. The correct sequence of this tryptic peptide (ALFHGR) was determined and reported in less than $15 \mathrm{~s}$ by using the default parameters. The failure to interpret the complete structure of the undigested peptide can readily be attributed to a lack of sequence ions derived from some amino acids. In short, the data did not completely document the structure. Analysis of the tandem data from the undigested peptide did. however, predict an internal arginine residue and predict the size of tryptic peptides to be obtained on digestion. Thus, while a complete answer was not obtainable from the CID spectrum, partial analysis did suggest a plan to complete the characterization of the peptide. It must be noted that determination of the test peptide sequence was primarily performed by manual analysis of the liquid secondary ion mass spectrum (LSIMS) and CID on both the whole and digested peptides. Automated integration of data obtained from initial fragmentation in the LSIMS into the sequencing process would prove to be a powerful approach. The simpler fragmentation patterns sometimes found in the LSIMS can lead to better restrictions on the identity of peaks also present in the CID spectrum. Further simplification occurs for the first and last amino acids in the sequence, as the high mass end of LSI mass spectra is not as populated by side chain losses as are CID spectra. One of the strongest features of our program is its ability to quickly identify reliable partial interpretations of data. Strategies for confident completion of sequence assignments can then be based on the weakest structural clues presented and ways available to improve confidence in those regions.

A single pass pattern recognition is used in the program described in this article. A promising alternative would be a progressive refining of ion labeling. This might work in the following fashion. The first pass step described above would detect candidate $y$-centers. Strongly represented $y$-centers that are not mutually exclusive would be identified. Mutually exclusive means either through coinclusion of peaks or by virtue of being unlinkable by an amino acid (such as centers $44 \mathrm{u}$ apart). The identities of the ions within these families would be fuxed as "known." A second pass of $y$-center detection would then not use the "known" ions in alternative contexts. This approach could eliminate some of the symmetry problems demonstrated in Figure 2 and thereby diminish the number of false $y$-centers considered during linking and sequence tracing.

It remains to understand and then use the more subtle patterns present in CID spectra of peptides. Some of the patterns that show the greatest chance of providing useful information involve the increase in abundance of certain sequence ion series depending on the positioning and relative pKas of basic side chains within the peptide being analyzed. An additional hope is to assign probabilities of observing certain ion types for cleavages between specific amino acid pairs. Further improvement in our ability to predict spectra based on sequence will be used to improve scoring. Accurate predictions will eventually result in scoring strategies based more on consistency with expectation, rather than relative strength of signal. We believe that this level of analysis will ultimately be needed to generate confidence scores for ranking peptide sequence matches in any meaningful way.

\section{Acknowledgment}

We are gratefully indebted to all the investigators in our lab who have shared their data prior to its publication. We are especially 
grateful to Kati Medzihradszky, whose expertise in manual analysis of CID spectra helped us in many ways, and to Fred Walls for acquiring most of the spectra used in this work. We acknowledge financial support from the National Institutes of Health National Center for Research Resources DRR RR01614 (to ALB) and the National Science Foundation Biological Instrumentation Program Grant DIR 8700766 (to ALB).

\section{References}

1. Aebersold, R.; Leavitt, J. Electrophovesis 1990, 11, 515-527.

2. Ullich, A.; Bell, J. R.; Chen, E. Y.; Herrera, R.; Petruzzelli, L. M.; Dull, T. J.; Gray, A.; Coussens, L.; Liao, Y.-C.; Tsubokawa, M.; Mason, A.; Seeburg, P. H.; Grunfeld, C.; Rosen, O. M.; Ramachandran, J. Nature 1985, 313, 756-761.

3. McGrath, M. E.; Hines, W. M.; Sakanar, J. A.; Fletterick, R. J.; Craik, C. S. J. Biol. Chem. 1991, 266, 6620-6625.

4. Anderegg, R. J.; Carr, S. A.; Huang, Y.; Hiipakka, R. A.; Chang, C.; Liao, S. Biochemistry 1988, 27, 4214-4221.

5. Biemann, K.; Gapp, F.; Seibl, J. J. Am. Chem. Soc. 1959, 81, 2274-2276

6. Carr, S. A.; Biemann, K. Meth. Enzymol. 1986, 106, 29-58.

7. Evans, S. Meth. Enzymol. 1990, 193, 61-86.

8. Ashcroft, A. E.; Derrick, P. J. In Mass Spectrometry of Peptides; Desiderio, D. M., Ed.; CRC: Boca Raton, FL, 1991; pp 121-138.

9. Hill, J. A.; Martin, 5. A.; Biller, J. E.; Biemann, K. Biomed. Environ. Mass. Spectrom. 1988, 17, 147-151.

10. Walls, F. C.; Baldwin, M. A.; Falick, A. M.; Gibson, B. W.; Kaur, S.; Maltby, D. A.; Gillece-Castro, B. L.; Medzihradszky, K. F.; Evans. S.; Burlingame. A. L. In Biological Mass Spectrometry; Burlingame, A. L.; McCloskey, J. A., Eds.; Elsevier: Amsterdam, 1990; pp 197-216.

11. Fenn, J. B. Science 1989, 246, 4926.

12. Loo, J. A.; Edmonds, C. G.; Smith, R. D. Science 1990, 248, 201.

13. Edmonds, C. G.; Smith, R. D. Meth. Enzymol. 1990, 193, 412-431.

14. Karas, M.; Hillenkamp, F. Anal. Chem. 1988, 60, 2299-2301.

15. Hillenkamp, F.; Karas, M. Meth. Enzymol. 1990. 193, 280-294.

16. Bean, M. F.; Carr, S. A.; Thorne, G. C.; Reilly, M. H.; Gaskell, S. J. Anal. Chem. 1991, 63, 1473-1481.

17. Alexander, A. J.: Thibault, P.; Boyd, R. K.; Curtis, J. M.; Rinehart, K. L. Int. J. Mass Spectrom. Ion Processes 1990, 98 , $107-134$.

18. Yates, J. R.; Griffin, P. R.; Mao, Y.; Hood, L. E.; Zhou, J. X.
Proceedings of the 37th ASMS Conference on Mass Spectrometry and Allied Topics; Nashville, TN, 1991; p 1221.

19. Burlingame, A. L.; Walls, F. C.; Falick, A.; Evans. S. M. Rapid Contmun. Mass Spectrom. 1990, 4, 447-448.

20. Ishikawa, K.; Niwa, Y. Biomed. Environ. Mass Spectrom. $1986,13,373-380$.

21. Klotz, A. V.; Thomas, B. A.; Glazer, A. N.; Blacher, R. W. Anal. Biochem. 1990, 186, 95-100.

22. Janes, S. M.; Mu, D.; Wemmer, D.; Smith, A. J.; Kaur, S.; Maltby, D.; Burlingame, A. L.; Klinman, J. P. Science 1990, 248, 981-987.

23. Hewick, R. M.; Hunkapiller, M. W.; Hood. L. E.; Dryer, W. J. J. Biol. Chem. 1981, 256, 7990-7997.

24. Johnson, R. S.; Biemann, K. Biomed. Environ. Mass Spectrom. 1989, 18, 945-957.

25. Lee, T. D.; Shively, J. E. Meth. Enzymol. 1990, 193, 361-373.

26. Roepstorff, P.; Fohlmann, J. Biomed. Mass Spectrom. 1984, 11, 601.; Biemann, K. Meth. Enzymol. 1990, 193, 886-887.

27. Martin, S. A.; Biemann, K. Int. I. Mass Spectrom. Ion Processes 1987, 78, 218-228.

28. Johnson, R. S.; Martin, S. A.; Biemann, K.; Stults, J. T.; Watson, J. T. Anal. Chem. 1987, 59, 2621-2625.

29. Johnson, R. S.; Martin, S. A.; Biemann, K. Int. J. Mass Spectrom. Ion Processes 1988, 86, 137-154

30. Stults, I. T.; Watson, J. T. Biomed. Environ. Mass Spectrom. 1987, 14, 583-586.

31. Falick, A. M.; Medzihradszky, K. F.; Hines, W. M.; Baldwin, M. A.; Gibson, B. W. In preparation.

32. Boyd, R. K. Int. J. Mass Spectrom. Ion Processes 1987, 75, 243-264.

33. Bartels, C. Biomed. Environ. Mass Spectrom. 1990, 19, $363-368$.

34. Zidarov, D.; Thibault, P.; Evans, M. J.; Bertrand, M. J. Biomed. Environ. Mass Spectrom. 1990, 19, 13-26.

35. Brozolo, F. R. D.; Odom, R. W.; Harrington, P. D. B.; Voorhees, K. J. J. Appl. Polymer Sci. 1990, 41, 1737-1752.

36. Gray, N. A. B.; Smith, D. H.; Varkony, T. H.; Carhart, R, E.; Buchanan, B. G. In Biochemical Applications of Mass Spectrometry, first suppl. volume; Waller, G. R.; Dermer, O. C., Eds.; Wiley-Interscience: New York, 1980; pp 125-149.

37. Morris, H. R. Nature 1980, 286, 447-452.

38. Wade, A. P.; Palmer, P. T.; Hart, K. J.; Enke, C. G. Anal. Chim. Acta 1988, 215, 169-186.

39. Medzihradszky, K. F.; Hall, S. C.; Maltby, D. A.; Hines, W.; Burlingame, A. L. In Techniques in Protein Chemistry II; Villafranca, J., Ed.; Harcourt Brace Jovanovich: San Diego, 1990; pp 435-440. 